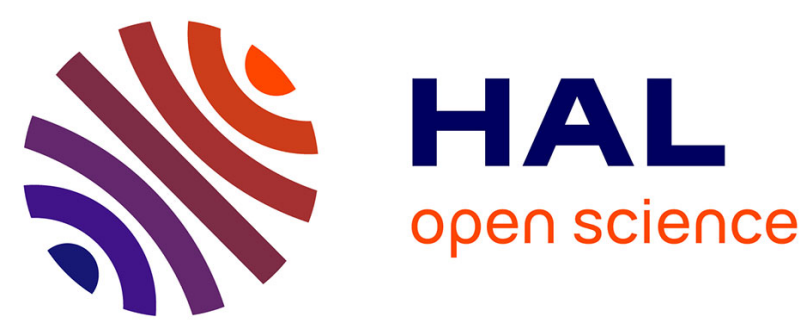

\title{
How to cope with unmeasurable premise variables in Takagi-Sugeno observer design: Dynamic extension approach
}

\author{
Dalil Ichalal, Benoît Marx, Saïd Mammar, Didier Maquin, José Ragot
}

\section{To cite this version:}

Dalil Ichalal, Benoît Marx, Saïd Mammar, Didier Maquin, José Ragot. How to cope with unmeasurable premise variables in Takagi-Sugeno observer design: Dynamic extension approach. Engineering Applications of Artificial Intelligence, 2018, 67, pp.430-435. 10.1016/j.engappai.2017.09.018 . hal01609899

\section{HAL Id: hal-01609899 \\ https://hal.science/hal-01609899}

Submitted on 17 Apr 2019

HAL is a multi-disciplinary open access archive for the deposit and dissemination of scientific research documents, whether they are published or not. The documents may come from teaching and research institutions in France or abroad, or from public or private research centers.
L'archive ouverte pluridisciplinaire HAL, est destinée au dépôt et à la diffusion de documents scientifiques de niveau recherche, publiés ou non, émanant des établissements d'enseignement et de recherche français ou étrangers, des laboratoires publics ou privés. 


\title{
How to cope with unmeasurable premise variables in Takagi-Sugeno observer design: Dynamic extension approach
}

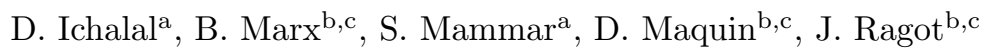 \\ ${ }^{a}$ Evry Val d'Essonne University, IBISC-Lab, 40, rue de Pelvoux, 91020, Evry \\ Courcouronnes, France (email: dalil.ichalal@ibisc.univ-evry.fr) \\ ${ }^{b}$ Université de Lorraine, CRAN, UMR 7039, 2 avenue de la Foret de Haye, TSA 60604, \\ 54518 Vandoeuvre-les-Nancy, France \\ ${ }^{c}$ CNRS, CRAN, UMR 7039, France
}

\begin{abstract}
In this paper, a new strategy to cope with unmeasurable premise variables in observer design for Takagi-Sugeno (TS) models is proposed. The guiding principles are the immersion techniques and auxiliary dynamics generation, allowing to immerge a given TS system with unmeasured state dependent weighting functions into a larger TS system with weighting functions depending only on measured variables. This result relaxes the strong conditions used in the design of observers for TS systems with unmeasurable premise variables. An example is provided to illustrate the performances of the proposed approach.
\end{abstract}

Keywords: Nonlinear estimation, Takagi-Sugeno systems, Immersion

\section{Introduction}

For economical or physical reasons, sensors cannot always be used to measure all the system states, thus observer design is a key point in process control or supervision. Since the seminal works of Luenberger (1971) or Kalman (1960), linear estimation has drawn a lot of attention. Unfortunately, the accurate description of a process generally results in a nonlinear model and nonlinear state estimation can only be solved for very specific model structures (triangular, linear with output injection, etc). An appealing way to generalize some linear tools to nonlinear systems is the TS approach proposed by Takagi and Sugeno (1985), that is based on an exact rewriting of the nonlinear model, at least on a compact set of the state space, thanks to the sector nonlinearity transformation (SNT) (Tanaka and Wang, 2001). Nevertheless, if the nonlinearity of the system involves the state variables, the premise variables (PV) used for the interpolation between the linear submodels are unmeasurable and the observer design is no longer a trivial task. Thus, in most of the proposed results, the PV are assumed to be known, even if it drastically reduces the validity of the TS approach. In 
the present paper observer design for TS systems with state dependent PV is addressed.

In the last fifteen years, the problem of state estimation of TS systems with state dependent PV has been investigated but it remains an open research problem. The first results, based on the Thau-Luenberger observer (Thau, 1973) and a Lipschitz assumption, were proposed by Bergsten et al. (2002) and then relaxed by Ichalal et al. (2010) or extended by Lendek et al. (2009) for TS cascaded systems. Unfortunately, the admissible Lipschitz constant ensuring the existence of a solution is often very small which limits the applicability of the approach. In order to avoid this limitation, a new approach based on the Differential Mean Value Theorem (DMVT) was given in (Ichalal et al. 2011). More recently, a new solution aims to reduce the conservativeness of the Lipschitz approach and the computational complexity of the DMVT approach, by leaving the asymptotic convergence and seeking to bound the estimation error within the $\mathcal{L}_{2}$ framework in (Ichalal et al. 2009 ) or with the quasi Inputto-State Stability (qISS) in (Ichalal et al., 2012).

Another way to generalize the numerous and efficient linear observer designs to nonlinear systems is to find coordinate transformations that transform the original nonlinear system into another one with a particular structure: a linear system modulo output injection (Krener and Isidori, 1983), a state affine system, a S-system (Savageau and Voit, 1987), a triangular system (Gauthier and Kupka, 2001), and then design the observer by mimicking the obtained structure. Among these techniques one should cite the output injection linearization, introduced by Krener and Isidori (1983) and successfully used by (Kazantzis and Kravaris, 1998, Besançon, 2007, 1999, Souleiman et al., 2003) and the immersion or dynamics extension introduced by Claude et al. (1983). Necessary and sufficient conditions to obtain a linear model by immersion have been given by Levine and Marino (1986). More recently, Besançon and Ticlea (2007) have pointed out the difficulty (or even the impossibility) to immerse a nonlinear system into a linear one and have proposed to generalize the target model structure from linear to state and input affine.

In the present paper, we propose to combine the two previously mentioned approaches. First, the original nonlinear system is transformed by immersion. The goal is to extend the state vector such that the original system is immersed into a larger state affine system or quasi-LPV (q-LPV) with nonlinearities depending only on measurable signals (inputs and outputs). Then, the SNT is applied on the extended system to obtain a TS system with measurable PV. Finally, the observer design is performed on the TS with measurable PV. Compared to existing works using immersion technique, the novelty is that the sought form, namely the q-LPV formalism, is more generic than the previous ones (e.g. linear with output injection, observable normal forms, polynomial systems, Ssystems). Moreover, in the proposed coordinate transformation the original state variables to estimate are the $n$ first components of the extended state vector. Consequently, no inverse transformation is needed to recover the original state variables.

The paper is organized as follows. in the second section some backgrounds 
on observer design for TS systems are given and the problem is stated. The third section is devoted to the main result of the paper: a new observer design algorithm. Simulations and comparisons with existing results are provided in the fourth section. Before concluding, a brief discussion is exposed.

\section{Preliminaries and problem statement}

Let us consider a nonlinear system defined by

$$
\left\{\begin{array}{l}
\dot{x}(t)=f(x(t), u(t)) \\
y(t)=C x(t)
\end{array}\right.
$$

where $x \in \mathbb{R}^{n}, u \in \mathbb{R}^{n_{u}}$ and $y \in \mathbb{R}^{n_{y}}$ are the system state, input and output vectors. The general goal is to design an observer providing a state estimate, denoted $\hat{x}(t)$, based on the knowledge of the system input and output.

With the SNT, (1) is written as a TS system defined by

$$
\left\{\begin{aligned}
\dot{x}(t) & =\sum_{i=1}^{r} h_{i}(\xi(t))\left(A_{i} x(t)+B_{i} u(t)\right) \\
y(t) & =C x(t)
\end{aligned}\right.
$$

where the nonnegative functions $h_{i}$, called the weighting functions, satisfy the convex sum property (i.e. their sum is equal to 1 , for all $t$ ) and depend on the so-called premise variable $\xi(t)$. If the PV is accessible, the following observer can be used

$$
\left\{\begin{array}{l}
\dot{\hat{x}}(t)=\sum_{i=1}^{r} h_{i}(\xi(t))\left(A_{i} \hat{x}(t)+B_{i} u(t)+L_{i}(y(t)-\hat{y}(t))\right) \\
\hat{y}(t)=C \hat{x}(t)
\end{array}\right.
$$

Since the system (2) and the observer (3) share the same PV, the state estimation error $e(t)=x(t)-\hat{x}(t)$ can easily be written as an autonomous TS system : $\dot{e}(t)=\sum_{i=1}^{r} h_{i}(\xi(t))\left(A_{i}-L_{i} C_{j}\right) e(t)$. Then, standard stability analysis results in sufficient LMI conditions to obtain the observer gains (Tanaka et al., 1998: Tanaka and Wang, 2001) that have been improved by using relaxed LMI conditions (Márquez et al., 2014, Guerra et al., 2015; González et al., 2016).

If the PV depend on the state and then are unmeasurable, it cannot be used in the observer and (3) cannot be constructed. In such a case, the observer does not share the same VP as the TS system (2) but only the estimated ones, as follows

$$
\left\{\begin{array}{l}
\dot{\hat{x}}(t)=\sum_{i=1}^{r} h_{i}(\hat{\xi}(t))\left(A_{i} \hat{x}(t)+B_{i} u(t)+L_{i}(y(t)-\hat{y}(t))\right) \\
\hat{y}(t)=C \hat{x}(t)
\end{array}\right.
$$

Consequently, the state estimation error cannot be written as an autonomous TS system anymore, but it can be turned into the following pseudo-disturbed TS system

$$
e(t)=\sum_{i=1}^{r} h_{i}(\hat{\xi}(t))\left(A_{i}-L_{i} C\right) e(t)+\delta(t)
$$


where the perturbation-like term $\delta(t)$ is given by

$$
\delta(t)=\sum_{i=1}^{r}\left(h_{i}(\xi)-h_{i}(\hat{\xi})\right)\left(A_{i} x(t)+B_{i} u(t)\right)
$$

It is then clear that, because of the $\delta(t)$ term, ensuring the stability of (5) is much more difficult than for an autonomous TS system and the conservative Lipschitz, DMVT or qISS approaches have to be used.

In the present paper, a new observer design is proposed in order to avoid the drawbacks of the Lipschitz, DMVT or qISS approaches by bridging the estimation of TS systems with unmeasurable PV to the one of TS systems with measurable PV. More precisely, before using the SNT on the nonlinear system (1), which generally gives rise to a TS system with unmeasurable PV, the immersion is used in order to express (1) as a q-LPV system with matrices depending only on measured variables. Then, the SNT can be used in order to exactly express the new system in TS form. Finally, since the PV of the obtained TS model are measurable, the classical observer (3) can be used. In addition, the proposed approach aims to generate new coordinates that contain the original state vector and then avoid tedious inverse transformation after immersion.

\section{Main result}

In order to link the design of TS observer with estimated PV (4) to the less conservative design of TS observer with measured PV (3), the following 3-step procedure is proposed.

Firstly, use auxiliary dynamics to define new coordinate and immerse the nonlinear system (1) into a q-LPV system whose parameters only depend on the measured signals $u(t)$ and $y(t)$

$$
\left\{\begin{array}{l}
\dot{z}(t)=A(u(t), y(t)) z(t)+B(u(t), y(t)) u(t)+\varphi(u(t), y(t)) \\
y(t)=\left[\begin{array}{ll}
C & 0
\end{array}\right] z(t)
\end{array}\right.
$$

Then, apply the SNT to the q-LPV system to obtain the following TS system with measured $\mathrm{PV}$, on the compact set $\mathcal{U} \times \mathcal{Y}$, where $u \in \mathcal{U}$ and $y \in \mathcal{Y}$

$$
\left\{\begin{array}{l}
\dot{z}(t)=\sum_{i=1}^{r} h_{i}(\xi(t))\left(A_{i} z(t)+B_{i} u(t)\right)+\varphi(u(t), y(t)) \\
y(t)=[C 0] z(t)
\end{array}\right.
$$

Finally, design the TS observer

$$
\left\{\begin{aligned}
\dot{\hat{z}}(t)= & \sum_{i=1}^{r} h_{i}(\xi(t))\left(A_{i} \hat{z}(t)+B_{i} u(t)+\varphi(u(t), y(t))\right) \\
& +L_{i}(y(t)-\hat{y}(t)) \\
\hat{y}(t)= & {\left[\begin{array}{ll}
C & 0
\end{array}\right] \hat{z}(t) }
\end{aligned}\right.
$$


The PV $\xi(t)$ of (8) and (9) only depends on measurable variables $y(t)$ and $u(t)$, then the dynamics of the extended state estimation error $e_{z}(t)=z(t)-\hat{z}(t)$, readily is an autonomous TS system with measurable PV. Thus, the complex observer design for TS systems with unmeasurable PV is avoided. Since the novelty mainly lies in the first step of the proposed procedure, this step is detailed hereafter.

\subsection{Auxiliary dynamics generation}

The technique consists in immersing the state space, $x \in \mathbb{R}^{n}$, of the original system (1) into a larger state space: $z \in \mathbb{R}^{N}$, with $N \geq n$. This transformation only affects the state space, but the input-output map of (1) is preserved. Indeed, this approach can be seen as the extension of the state vector with new variables coming from the system nonlinearities. The coordinate transformation procedure is the following.

Step 1.1. The $n$ first new variables are defined by the original state variables: $z_{i}=x_{i}$, for $i=1, \ldots, n$.

Step 1.2. For $k=1, \ldots, n$, write the time derivative of $z_{k}(t)$ according to (1) as the following q-LPV system with additive nonlinear term $\varphi(u, y)$

$$
\begin{aligned}
\dot{z}_{k}(t)= & \sum_{i=1}^{n} a_{k, i}(u(t), y(t)) z_{i}(t) \\
& +\sum_{i=n+1}^{\ell} a_{k, i}(u(t), y(t)) z_{i}(t)+\varphi_{k}(u(t), y(t))
\end{aligned}
$$

if $a_{k, i}=0$ for $i>n$, no new variables is needed and the algorithm ends. If new variables $z_{i}$ with $i>n$ are needed, go to step 1.3.

Step 1.3. For each new variable, compute its time derivative according to (1), separate all the functions depending only on measured variables $y$ and $u$ and define the remaining nonlinear functions as new variables $z_{k}, k>n$. By differentiating these variables it follows

$$
\begin{aligned}
\dot{z}_{k}(t)= & \sum_{i=1}^{k} a_{k, i}(u(t), y(t)) z_{i}(t) \\
& +\sum_{i=k+1}^{\ell} a_{k, i}(u(t), y(t)) z_{i}(t)+\varphi_{k}(u(t), y(t))
\end{aligned}
$$

where $z_{i}, i=k+1, \ldots, \ell$ denote other defined new variables.

Step 1.4. If the time derivative of the $k$ first state variables, $\dot{z}_{i}(t)$ for $i=$ $1, \ldots, k$, depend on new variables $z_{\ell}$ with $\ell>k$, then go to step 1.2. Else : algorithm ends.

This procedure is similar to the one presented in (Ticlea, 2006) except for the first step. In the first step of (Ticlea, 2006), the first variables are initialized as 
the $n_{y}$ system outputs while in the present approach the $n$ system states of (1) are used. This avoids the computation of the inverse transformation. A similar idea was introduced by Bernard et al. (2015) for high gain observer design by Jacobian completion.

Moreover, in the existing results using immersion in nonlinear estimation, the target model took particular forms such (e.g. linear observable form with output injection, observable state affine model with output injection, S-systems, etc). Unfortunately, the computation of the coordinate transformation may be very difficult for such target models. The proposed algorithm aims to transform the original system into a more generic form (7) and then relaxes the search of the adequate transformation. One should also note that this coordinate transformation is not unique, thus transformation that preserves the properties of the original system (e.g. observability, detectability) should be preferred.

Remark 1. The previous result can be extended to nonlinear systems affected by unknown inputs (UI) denoted $d(t)$

$$
\left\{\begin{array}{l}
\dot{x}(t)=f(x(t), u(t))+l(x(t)) d(t) \\
y(t)=C x(t)
\end{array}\right.
$$

If the UI is constant, or more generally a time polynomial function satisfying $d^{(j)}(t)=0$ for a positive integer $j$, the system state can be augmented with the $j-1$ first derivatives of the UI $\tilde{x}=\left[\begin{array}{lll}x^{T} & d^{T} \ldots\left(d^{(j-1)}\right)^{T}\end{array}\right]^{T}$ and the previous algorithm can be applied. If the UI is not a time polynomial, the obtained $q-L P V$ system is in the form:

$$
\left\{\begin{array}{l}
\dot{x}(t)=A(u(t), y(t)) x(t)+B(u(t), y(t)) u(t)+E(u(t), y(t)) d(t) \\
y(t)=C x(t)
\end{array}\right.
$$

and it may be possible to design decoupling UI observers after obtaining the TS model with measurable PV. Classical UI Observer can be designed provided the following condition holds: $\operatorname{rank}(C E(u, y))=\operatorname{rank}(E(u, y)), \forall y \in \mathcal{Y}, \quad \forall u \in \mathcal{U}$. If this condition is not satisfied, the design proposed by Ichalal and Mammar (2015) can be used. It consists in using some time derivatives of the output and the input up to finite orders.

\section{Example and comparisons}

In this section, the proposed approach is compared to the existing approaches on the Lorenz system (widely used in signal processing for secured communication, see Cuomo et al. (1993)) defined by

$$
\left\{\begin{array}{l}
\dot{x}_{1}(t)=a\left(x_{1}(t)-x_{2}(t)\right) \\
\dot{x}_{2}(t)=b x_{1}(t)-x_{2}(t)-x_{1}(t) x_{3}(t) \\
\dot{x}_{3}(t)=c x_{3}(t)+x_{1}(t) x_{2}(t) \\
y(t)=x_{2}(t)
\end{array}\right.
$$

where $a=-10, b=28$ and $c=-\frac{8}{3}$. 


\subsection{Existing approaches}

In this first subsection, some existing observer design approaches are applied, namely the Lipschitz, the DMVT and q-ISS are here detailed.

Lipschitz approach. In the so-called Lipschitz approach, proposed in (Bergsten et al., 2002, Lendek et al. 2009), the perturbation-like term is assumed to be Lipschitz in the state estimation error. The system (14) is expressed as the following quasi-LPV form to apply the SNT

$$
\left\{\begin{aligned}
\dot{x}(t) & =\left[\begin{array}{ccc}
a & -a & 0 \\
b & -1 & -x_{1}(t) \\
0 & x_{1}(t) & c
\end{array}\right] x(t) \\
y(t) & =\left[\begin{array}{ccc}
0 & 1 & 0
\end{array}\right] x(t)
\end{aligned}\right.
$$

Defining $\xi(t)=x_{1}(t)$ and assuming that $\xi_{\min } \leq \xi(t) \leq \xi_{\max }$, the obtained TS system is

$$
\dot{x}(t)=\sum_{i=1}^{2} h_{i}(\xi(t)) A_{i} x(t)
$$

where $h_{1}(\xi(t))=\frac{\xi(t)-\xi_{\min }}{\xi_{\max }-\xi_{\min }}, h_{2}(\xi(t))=\frac{\xi_{\max }-\xi(t)}{\xi_{\max }-\xi_{\min }}$ and

$$
A_{1}=\left[\begin{array}{ccc}
a & -a & 0 \\
b & -1 & -\xi_{\max } \\
0 & \xi_{\max } & c
\end{array}\right], A_{2}=\left[\begin{array}{ccc}
a & -a & 0 \\
b & -1 & -\xi_{\min } \\
0 & \xi_{\min } & c
\end{array}\right]
$$

The Lipschitz observer is (4) with $B_{i}=0$, where the PV is the estimated first state $\hat{x}_{1}(t)$. The state estimation error $e(t)$ obeys to (5) where the perturbationlike term (6) is defined by

$$
\delta(t)=\left[\begin{array}{ccc}
0 & 0 & 0 \\
0 & 0 & -(\xi(t)-\hat{\xi}(t)) \\
0 & \xi(t)-\hat{\xi}(t) & 0
\end{array}\right] x(t)
$$

With $\xi(t)=x_{1}(t)$, it readily follows that $|\xi(t)-\hat{\xi}(t)| \leq\|e(t)\|$. The state variables of the Lorenz system are bounded by $\left|x_{2}(t)\right|<\sigma_{2}$ and $\left|x_{3}(t)\right|<\sigma_{3}$ with $\sigma_{3}>\sigma_{2}$. Thus the term $\delta(t)$ can be bounded as follows

$$
\|\delta(t)\|<\gamma\|e(t)\|
$$

where $\gamma=\sigma_{3}$ is the Lipschitz constant of $\delta(t)$. The bounds of the state variable $x_{1}(t)$, obtained in simulation for $x(0)=\left[\begin{array}{lll}0 & 0 & 0\end{array}\right]^{T}$, allow to chose the following bounds on the PV: $\xi_{\min }=-20$ and $\xi_{\max }=20$ and the real Lipschitz constant is $\gamma=48$. According to these assumptions, the sufficient observer existence conditions are given by the set of 3 LMIs of the Theorem 1 in (Bergsten et al. 2002). In this procedure, the maximum admissible Lipschitz constant $\gamma^{*}$ can be computed in the LMI optimization. Using the YALMIP and SEDUMI solver 
(Lofberg, 2004), it is found to be $\gamma^{*}=2.6667$. Since the real Lipschitz constant $\gamma$ of the system is greater that the maximum admissible one $\gamma^{*}$, no solution can be found and the observer design fails.

Differential Mean Value Theorem approach. Following the approach proposed by Ichalal et al. (2011), the TS model obtained in the previous section is used. The difference compared to the Lipschitz approach is in the way of handling the term $\delta(t)$ (17). This term can be expressed as follows

$$
\delta(t)=\left[\begin{array}{c}
0 \\
-x_{3}(t) \\
x_{2}(t)
\end{array}\right](\xi(t)-\hat{\xi}(t))
$$

Since the nonlinear function $\xi(t)=x_{1}(x)$ is differentiable and denoting $\hat{\xi}(t)=$ $\hat{x}_{1}(t)$, the DMVT states the existence of a constant vector $\left.c \in\right] x, \hat{x}[$ such that

$$
\xi(t)-\hat{\xi}(t)=\xi(x)-\xi(\hat{x})=\frac{\partial \xi}{\partial x^{T}}(c)(x-\hat{x})
$$

Since $\xi(t)=x_{1}(t)$, it follows that $\frac{\partial \xi}{\partial x^{T}}=\left[\begin{array}{lll}1 & 0 & 0\end{array}\right]$ and $\delta(t)$ becomes

$$
\delta(t)=\left[\begin{array}{ccc}
0 & 0 & 0 \\
-x_{3}(t) & 0 & 0 \\
x_{2}(t) & 0 & 0
\end{array}\right] e(t)
$$

The state variables $x_{2}(t)$ and $x_{3}(t)$ are bounded, thus the sector nonlinearity approach can be applied to 21 to obtain the following TS writing of $\delta(t)$

$$
\delta(t)=\sum_{j=1}^{4} v_{j}\left(x_{2}(t), x_{3}(t)\right) \mathcal{A}_{j} e(t)
$$

and the state estimation error dynamic (5) becomes an autonomous TS system

$$
\dot{e}(t)=\sum_{i=1}^{2} \sum_{j=1}^{4} h_{i}(\hat{\xi}(t)) v_{j}\left(x_{2}(t), x_{3}(t)\right)\left(A_{i}+\mathcal{A}_{j}-L_{i} C\right) e(t)
$$

Using a quadratic Lyapunov function, the stability analysis of 23) leads to the following LMI conditions, with $P=P^{T}>0$

$$
\left(A_{i}+\mathcal{A}_{j}\right)^{T} P+P\left(A_{i}+\mathcal{A}_{j}\right)-C^{T} K_{i}^{T}-K_{i} C<0
$$

for $i=1,2$ and $j=1, \ldots, 4$. The observer gains are given by $L_{i}=P^{-1} K_{i}$. Considering the previous numerical values: $-20 \leq x_{1} \leq 20,-30 \leq x_{2} \leq 30$ and $0 \leq x_{3} \leq 50$, a solution to the set of 8 LMIs $(24)$ is obtained and the observer is designed. Even if the compact set $\mathcal{X}$ is enlarged, a solution is found and thus outperforms the Lipschitz approach. 
Quasi-ISS approach In the quasi-ISS approach (Ichalal et al., 2012), the same observer (4) is considered. The difference with the Lipschitz and DMVT approaches is that the term $\delta(t)$ is considered as an external bounded perturbation. Following (Ichalal et al., 2012), a solution ensuring ISS is found by solving 3 LMIs.

\subsection{Proposed approach with auxiliary dynamics}

Before applying the SNT to the original system (14), the proposed algorithm is first applied.

Step 1.1. Define the new variables $z_{i}(t)=x_{i}(t)$, for $i=1, \ldots, 3$.

Step 1.2. The time derivatives of the new variables are

$$
\left\{\begin{array}{l}
\dot{z}_{1}(t)=a\left(z_{1}(t)-z_{2}(t)\right) \\
\dot{z}_{2}(t)=b z_{1}(t)-z_{2}(t)-z_{1}(t) z_{3}(t) \\
\dot{z}_{3}(t)=c z_{3}(t)+z_{1}(t) z_{2}(t)
\end{array}\right.
$$

Two nonlinearities $z_{1}(t) z_{3}(t)$ and $z_{1}(t) z_{2}(t)$ appear in (25). The second one is already in the adequate form i.e. $y(t) z_{1}(t)$, but, the first one depends on unknown state variables. A new variable is defined by $z_{4}(t)=z_{1}(t) z_{3}(t)$.

Step 1.3. The time derivative of $z_{4}(t)$ is

$$
\dot{z}_{4}(t)=(a+c) z_{4}(t)-a y(t) z_{3}(t)+y(t) z_{1}^{2}(t)
$$

In $(26)$, a new nonlinearity appears and defines a new variable $z_{5}(t)=z_{1}^{2}(t)$.

Step 1.3. The time derivative of $z_{5}(t)$ is

$$
\dot{z}_{5}(t)=2 a z_{5}(t)-2 a y(t) z_{1}(t)
$$

Step 1.4. No new variables need to be defined. End.

With the proposed coordinate change $z_{1}(t)=x_{1}(t), z_{2}(t)=x_{2}(t), z_{3}(t)=$ $x_{3}(t), z_{4}(t)=x_{1}(t) x_{3}(t)$ and $z_{5}(t)=x_{1}^{2}(t)$, the original system (14) can be immersed into the larger 77 with $B(u(t), y(t))=0, \varphi(u(t), y(t))=0, C=\left[\begin{array}{lll}0 & 1 & 0\end{array}\right]$ and

$$
A(u(t), y(t))=\left[\begin{array}{ccccc}
-10 & 10 & 0 & 0 & 0 \\
28 & -1 & 0 & -1 & 0 \\
y(t) & 0 & -\frac{8}{3} & 0 & 0 \\
0 & 0 & 10 y(t) & -\frac{38}{3} & y(t) \\
20 y(t) & 0 & 0 & 0 & -20
\end{array}\right]
$$

where $A(u(t), y(t))$ only depends on the measured variable $y(t)$. Since $\xi_{\text {min }} \leq$ $y(t) \leq \xi_{\max }$, the following TS system is obtained.

$$
\left\{\begin{aligned}
\dot{z}(t) & =\sum_{i=1}^{2} h_{i}(y) A_{i} z(t) \\
y(t) & =C z(t)
\end{aligned}\right.
$$




$$
\begin{gathered}
\text { with } h_{1}(y(t))=\frac{y(t)-\xi_{\min }}{\xi_{\max }-\xi_{\min }}, h_{2}(y(t))=\frac{\xi_{\max }-y(t)}{\xi_{\max }-\xi_{\min }} \text { and } \\
A_{1}=\left[\begin{array}{ccccc}
-10 & 10 & 0 & 0 & 0 \\
28 & -1 & 0 & -1 & 0 \\
y_{\max } & 0 & -\frac{8}{3} & 0 & 0 \\
0 & 0 & 10 y_{\max } & -\frac{38}{3} & y_{\max } \\
20 y_{\max } & 0 & 0 & 0 & -20
\end{array}\right] \\
A_{2}=\left[\begin{array}{ccccc}
-10 & 10 & 0 & 0 & 0 \\
28 & -1 & 0 & -1 & 0 \\
y_{\min } & 0 & -\frac{8}{3} & 0 & 0 \\
0 & 0 & 10 y_{\min } & -\frac{38}{3} & y_{\min } \\
20 y_{\min } & 0 & 0 & 0 & -20
\end{array}\right]
\end{gathered}
$$

Note that 29 is equivalent to 14 in a compact set of the state space. The design of the TS observer (9) is now trivial by classical LMI optimization. Since, the extended state estimation error $e_{z}(t)=z(t)-\hat{z}(t)$ obeys to

$$
\dot{e}_{z}(t)=\sum_{i=1}^{2} h_{i}(y(t))\left(A_{i}-L_{i} C\right) e_{z}(t)
$$

its convergence to zero is ensured by solving the two LMIs

$$
P A_{i}-K_{i} C+A_{i}^{T} P-C^{T} K_{i}<0, i=1,2
$$

and the observer gains are given by $L_{i}=P^{-1} K_{i}$ (Tanaka and Wang, 2001). Note that, once $z(t)$ is estimated, the original state estimation directly follows from $\hat{x}_{i}(t)=\hat{z}_{i}(t)$, for $i=1, \ldots, 3$, without any inverse transformation. Compared to the Lipschitz-based approach, the present approach avoids the computation of the Lipschitz constant and reduces the conservatism related to the corresponding LMIs.

Using the previously given numerical values, the LMIs 32 are solved and the observer gains are given by

$$
L_{1}=10^{3}\left[\begin{array}{c}
-0.129 \\
0.1817 \\
0.0125 \\
-9.841 \\
0.2234
\end{array}\right], \quad L_{2}=10^{3}\left[\begin{array}{c}
-0.129 \\
0.1817 \\
-0.0125 \\
-9.840 \\
-0.2234
\end{array}\right]
$$

The Figure 1illustrates the asymptotic state estimation and the Figure 2 depicts the state estimation errors.

It can be seen that the proposed approach based on auxiliary dynamics outperforms the three classical approaches (Lipschitz, MVT, qISS). In terms of numerical complexity, only 2 LMIs are needed. In addition, asymptotic convergence is obtained compared to the qISS approach where only bounded error is guaranteed. Finally, in the previous approaches, high gains may be obtained since the objective is to counteract the nonlinear term $\delta(t)$ while in the proposed approach the gains remain small since no $\delta(t)$ needs to be counteracted. 

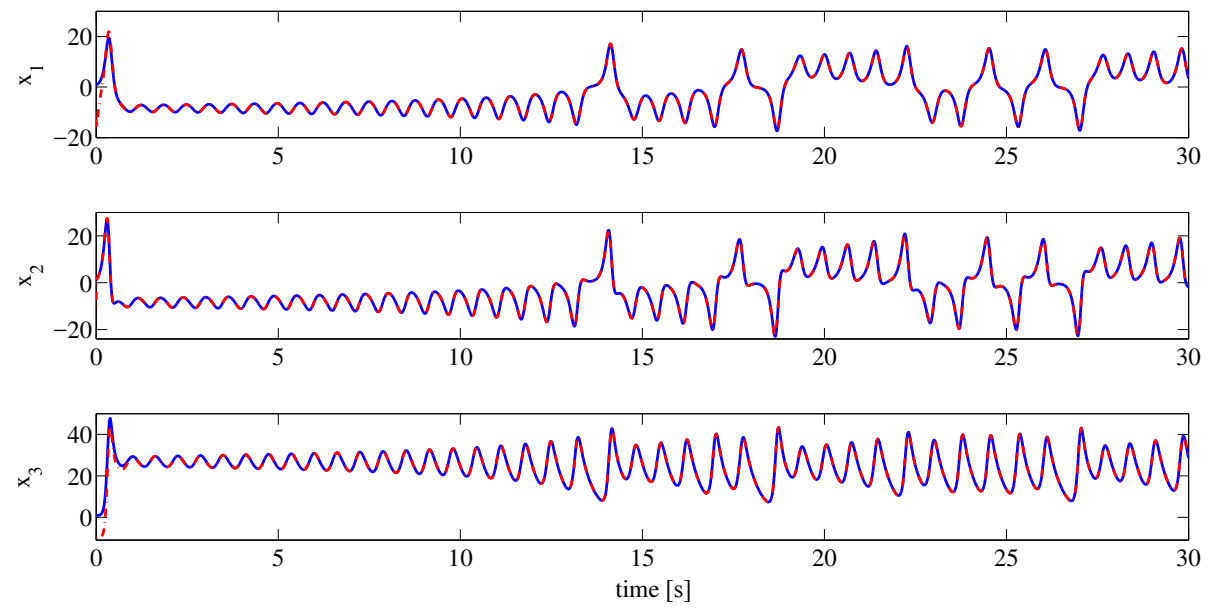

Figure 1: Original state variables (blue lines) and estimated ones (red lines)

\section{Discussion}

The proposed result aims to extend the system dynamics in order to obtain a TS model with measurable PV. However, the transformation presented in the algorithm may fail to provide the adequate system due to an infinite number of iterations. In order to illustrate this issue, let us consider the following system

$$
\left\{\begin{aligned}
\dot{x}_{1}(t) & =x_{1}(t) x_{2}(t) \\
\dot{x}_{2}(t) & =-x_{1}(t)-x_{2}^{2}(t) \\
y(t) & =x_{1}(t)
\end{aligned}\right.
$$

It is clear that directly applying the SNT inevitably leads to a TS system with a PV depending on the unmeasured state $x_{2}(t)$. Unfortunately, the proposed procedure also fails to provide a TS system with measurable PV. Indeed, the number of iterations is infinite meaning that the algorithm does not converge. The first step of the algorithm gives $z_{1}(t)=x_{1}(t)$ and $z_{2}(t)=x_{2}(t)$. The time derivative of $z_{1}(t)$ is under the form $\dot{z}_{1}(t)=f_{1}(y(t)) z(t)$ since it is given by

$$
\dot{z}_{1}(t)=y(t) z_{2}(t)
$$

Considering the second nonlinearity, the algorithm suggests to define the new variable $z_{3}(t)=z_{2}^{2}(t)$ and its time derivative is

$$
\dot{z}_{3}(t)=-2 z_{1}(t) z_{2}(t)-2 z_{2}^{3}(t)=-2 y(t) z_{2}(t)-2 z_{2}^{3}(t)
$$

Since a new nonlinearity appears in (35), the new variable $z_{4}(t)=z_{2}^{3}(t)$ is defined and its time derivative involves a new nonlinearity $z_{2}^{4}(t) \ldots$ and so on. Then the algorithm does not stop and fails to provide a solution. Some tougher new variable definitions may be used (here, defining $z_{3}(t)=z_{1}^{2}(t) z_{2}^{2}(t)$ leads to 

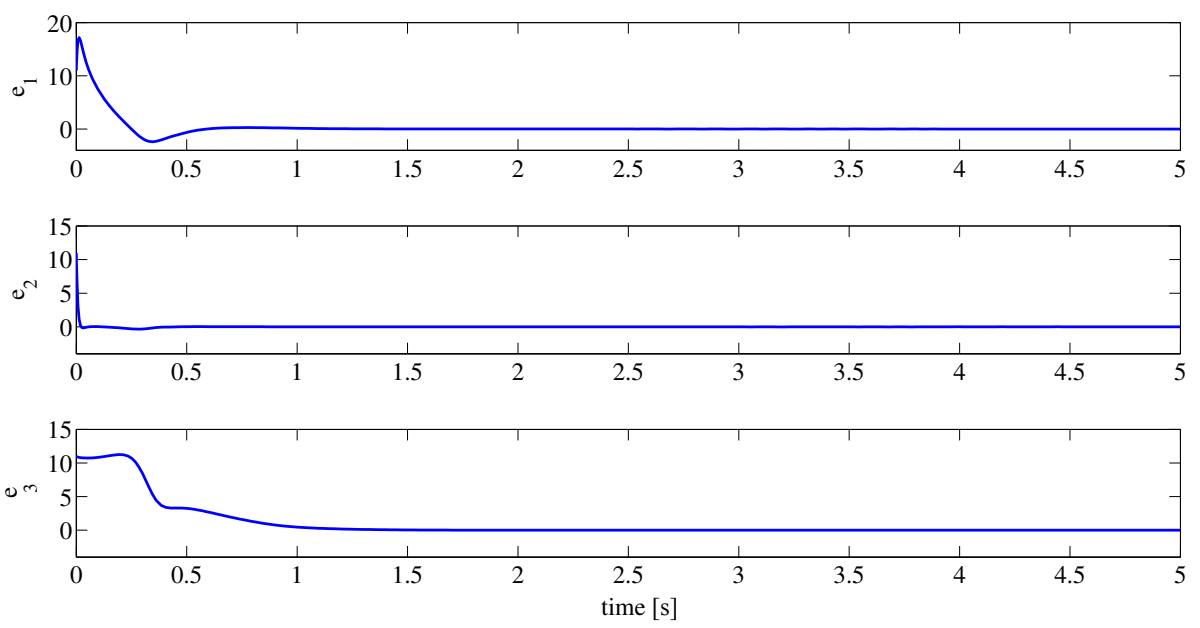

Figure 2: State estimation errors

$\dot{z}_{3}(t)=-2 y^{3}(t) z_{2}(t)$ and a solution is obtained with $N=3$ ) but they should be adapted to each particular case and then is out of the scope of the present study.

One may have noticed that the Lorenz system is a polynomial one Savageau and Voit (1987) and may know that the SoS (Sum-of-Squares) approach initiated by Tanaka et al. (2009); Narimani and Lam (2010) is widely used but unfortunately the case of unmeasurable premise variables is not treated in most of the existing works on observer design Seo et al. (2011); Han et al. (2017), only Sala et al. (2011) considered this case but proposed a local result.

\section{Conclusion}

In the present paper, the problem of observer design for nonlinear systems via TS systems is investigated. It is shown that using auxiliary dynamics and immersion techniques before the transformation of the original nonlinear system into a TS one is interesting to avoid state dependent premise variables and thus ease the observer design. The proposed algorithm consists in immersing the original nonlinear system in a larger system while preserving the input-output map. Then applying the sector nonlinearity transformation, the obtained system is transformed in TS system with measurable premise variables. The provided example clearly illustrates the effectiveness of the proposed approach.

\section{References}

Bergsten, P., Palm, R., Driankov, D., 2002. Observers for Takagi-Sugeno fuzzy systems. IEEE Transactions on Systems, Man, and Cybernetics - Part B: Cybernetics 32, 114-121. 
Bernard, P., Andrieu, V., Praly, L., 2015. Nonlinear observer in the original coordinates with diffeomorphism extension and Jacobian completion. ArXiv e-prints arXiv: 1509.04865.

Besançon, G., 1999. On output transformations for state linearization up to output injection. IEEE Transactions on Automatic Control, 44, 1975-1981.

Besançon, G., 2007. Nonlinear observers and applications. Lecture Notes In Control And Information Sciences (LNCIS), Springer.

Besançon, G., Ticlea, A., 2007. An immersion-based observer design for rankobservable nonlinear systems. IEEE Transactions on Automatic Control 52, $83-88$.

Claude, D., Fliess, M., Isidori, A., 1983. Immersion directe et par bouclage, d'un système non linéaire dans un linéaire. Comptes Rendus des Séances de l'Académie des Sciences de Paris. Série 1, Mathématique 296, 237-240.

Cuomo, K., Oppenheim, A., Strogatz, S., 1993. Synchronization of Lorenz-based chaotic circuits with applications to communications. IEEE Transactions on Circuits and Systems II: Analog and Digital Signal Processing 40, 626-633.

Gauthier, J.P., Kupka, I., 2001. Deterministic Observation Theory and Applications. Cambridge University Press.

González, T., Márquez, R., Bernal, M., Guerra, T.M., 2016. Nonquadratic controller and observer design for continuous ts models: A discrete-inspired solution. International Journal of Fuzzy Systems 18, 1-14.

Guerra, T., Estrada-Manzo, V., Lendek, Z., 2015. Observer design for TakagiSugeno descriptor models: An LMI approach. Automatica 52, 154-159.

Han, H., Chen, J., KarimChen, J., 2017. State and disturbance observers-based polynomial fuzzy controller. Information Sciences 382, 38-59.

Ichalal, D., Arioui, H., Mammar, S., 2011. Observer design for two-wheeled vehicle: A Takagi-Sugeno approach with unmeasurable premise variables, in: 19th Mediterranean Conference on Control Automation (MED), Corfu, Greece. pp. 934-939.

Ichalal, D., Mammar, S., 2015. On unknown input observers for LPV systems. IEEE Transactions on Industrial Electronics 62, 5870-5880.

Ichalal, D., Marx, B., Ragot, J., Maquin, D., 2009. State and unknown input estimation for nonlinear systems described by Takagi-Sugeno models with unmeasurable premise variables, in: 17th Mediterranean Conference on Control and Automation, MED'09, Thessaloniki, Greece. pp. 217-222.

Ichalal, D., Marx, B., Ragot, J., Maquin, D., 2010. State estimation of TakagiSugeno systems with unmeasurable premise variables. IET Control Theory \& Applications 4, 897-908. 
Ichalal, D., Marx, B., Ragot, J., Maquin, D., 2012. Advances in observer design for Takagi-Sugeno systems with unmeasurable premise variables, in: 20th Mediterranean Conference on Control Automation (MED), pp. 848-853.

Kalman, R., 1960. A new approach to linear filtering and prediction problems. Transactions of the ASME - Journal of Basic Engineering 82, 35-45.

Kazantzis, N., Kravaris, C., 1998. Nonlinear observer design using Lyapunov's auxiliary theorem. Systems \& Control Letters 34, 241-247.

Krener, A., Isidori, A., 1983. Linearization by output injection and nonlinear observers. Systems \& Control Letters 3, 47-52.

Lendek, Z., Babuska, R., De Schutter, B., 2009. Stability of cascaded fuzzy systems and observers. IEEE Transactions on Fuzzy Systems 17, 641 -653.

Levine, J., Marino, R., 1986. Nonlinear system immersion, observers and finite dimensional filters. Systems \& Control Letters 7, 133-142.

Lofberg, J., 2004. YALMIP : a toolbox for modeling and optimization in MATLAB, in: IEEE International Conference on Robotics and Automation, pp. $284-289$.

Luenberger, D., 1971. An introduction to observers. IEEE Transactions on Automatic Control 16, 596-602.

Márquez, R., Guerra, T.M., Kruszewski, A., Bernal, M., 2014. Decoupled nested LMI conditions for Takagi-Sugeno observer design, in: IFAC World Congress 2014, pp. 7994-7999.

Narimani, M., Lam, H., 2010. Sos-based stability analysis of polynomial fuzzymodel-based control systems via polynomial membership functions. IEEE Transactions on Fuzzy Systems 18, 862-871.

Sala, A., Pitarch, J., Bernal, M., Jaadari, A., Guerra, T., 2011. Fuzzy polynomial observers. IFAC World Congress .

Savageau, M., Voit, E., 1987. Recasting nonlinear differential equations as Ssystems: a canonical nonlinear form. Mathematical biosciences 87, 83-115.

Seo, T., Ohtake, H., Tanaka, K., Chen, Y., Wang, H., 2011. A polynomial observer design for a wider class of polynomial fuzzy systems, in: 2011 IEEE International Conference on Fuzzy Systems (FUZZ-IEEE 2011), pp. 13051311.

Souleiman, I., Glumineau, A., Schreier, G., 2003. Direct transformation of nonlinear systems into state affine MISO form for observer design. IEEE Transactions on Automatic Control 48, 2191-2196. 
Takagi, T., Sugeno, M., 1985. Fuzzy identification of systems and its applications to modeling and control. IEEE Transactions on Systems, Man, and Cybernetics 15, 116-132.

Tanaka, K., Ikeda, T., Wang, H., 1998. Fuzzy regulators and fuzzy observers: Relaxed stability conditions and LMI-based designs. IEEE Transactions on Fuzzy Systems 6, 250-265.

Tanaka, K., Wang, H., 2001. Fuzzy Control Systems Design and Analysis: A Linear Matrix Inequality Approach. John Wiley and Sons.

Tanaka, K., Yoshida, H., Othake, H., Wang, H., 2009. A sum-of-squares approach to modeling ans control of nonlinear dynamical systems with polynomial fuzzy systems. IEEE Transactions on Fuzzy Systems 17, 911-922.

Thau, F., 1973. Observing the state of non-linear dynamic systems. International Journal of Control 18, 471-479.

Ticlea, A., 2006. Techniques d'immersion pour l'estimation non linéaire : Application aux systèmes de puissances. Ph.D. thesis. Institut National Polytechnique de Grenoble (INPG). Grenoble, France. 